cemoti $\begin{aligned} & \text { Cahiers d'études sur la Méditerranée } \\ & \text { orientale et le monde turco-iranien }\end{aligned}$

$24 \mid 1997$

Métropoles et métropolisation

\title{
D’Athènes à Tachkent, métropoles et espaces métropolises
}

Marcel BAZIN

\section{(2) OpenEdition \\ Journals}

Édition électronique

URL : http://journals.openedition.org/cemoti/1455

DOI : $10.4000 /$ cemoti. 1455

ISSN : $1777-5396$

Éditeur

AFEMOTI

Édition imprimée

Date de publication : 1 juin 1997

ISSN : 0764-9878

Référence électronique

Marcel BAZIN, "D'Athènes à Tachkent, métropoles et espaces métropolises », Cahiers d'études sur la Méditerranée orientale et le monde turco-iranien [En ligne], 24 | 1997, mis en ligne le 28 février 2005, consulté le 08 septembre 2020. URL : http://journals.openedition.org/cemoti/1455 ; DOI : https:// doi.org/10.4000/cemoti. 1455

Ce document a été généré automatiquement le 8 septembre 2020

Tous droits réservés 


\title{
D’Athènes à Tachkent, métropoles et espaces métropolises
}

\author{
Marcel BAZIN
}

1 Partout dans le monde, le processus d'urbanisation semble connaître une accélération qui en changerait la nature, ou au moins le degré, en favorisant la concentration de la population dans les plus grandes villes, les seules à pouvoir cristalliser les fonctions de plus haut niveau : institutions politiques nationales et internationales, sièges sociaux des grandes firmes dites "multinationales", bourses des valeurs, équipements culturels de renommée mondiale etc... L'usage de ces adjectifs montre tout de suite le lien, que Jacques Lévy et Guy Burgel soulignent dans leurs contributions respectives à ce volume, entre la référence privilégiée à l'échelle mondiale, la mondialisation ${ }^{1}$, et ce nouveau stade de la croissance urbaine qu'on s'accorde à nommer la métropolisation ${ }^{2}$. Pierre Veltz a particulièrement insisté sur la liaison entre ces deux phénomènes en dépeignant l'économie mondialisée comme une « économie d'archipel» concentrant la richesse, le pouvoir et l'innovation technologique dans un petit nombre de grandes agglomérations émergeant d'un tissu interstitiel amorphe ${ }^{3}$. Si Saskia Sassen va plus loin encore en proposant le terme de "cité globale» (global city) 4 pour désigner une agglomération géante qui serait à la fois le résultat et l'instrument de la globalisation, et ne reconnaît ce titre qu'à New York, Londres et Tokyo, soit une seule cité globale pour chacun des trois pôles de la Triade qui mène le monde, Amérique du Nord, Europe occidentale et Japon, d'autres auteurs insistent sur le caractère beaucoup plus général de cette évolution, « orée d'un nouveau cycle long » qui réunit les grandes villes et les espaces environnants dans un processus de métapolisation pour François Ascher ${ }^{5}$ ou de métropolisation partagée pour Jacques Lévy ${ }^{6}$. Nous avons demandé à ce dernier d'apporter dans ce volume son éclairage théorique non seulement sur le phénomène de métropolisation, mais sur le fait urbain lui-même dont la métropolisation est en quelque sorte l'exacerbation.

2 Dans l'ensemble, ces réflexions sur la métropolisation sont nées à la fois dans et à propos des pays industriels - ne faudrait-il pas dire plus exactement aujourd'hui « postindustriels »? - les plus développés. Dans quelle mesure s'appliquent-elles au domaine 
d'étude des CEMOTI ? Notre objectif, en lançant l'élaboration de ce numéro, a été de fournir quelques éléments de réponse à cette question.

3 L'aire qui va de la Méditerranée orientale à l'Asie centrale appartient au "monde multipériphérique » que Jean Domingo et Bernard Elissalde ${ }^{7}$ décrivent autour de "l'économie-monde polycentrique de la Triade». Elle part des marges du pôle européen de la Triade, auquel la Grèce est rattachée institutionnellement, et présente un " gradient de développement » marqué à mesure qu'on s'éloigne vers l'est jusqu'en Afghanistan, un des pays les plus pauvres du monde. La décroissance des niveaux de développement suivant la séquence Grèce - Turquie de l'Ouest - Turquie de l'Est - Iran Afghanistan, d'une régularité caricaturale, semble illustrer parfaitement le schéma théorique d'auréoles concentriques de développement autour de l'Europe occidentale. Mais des anomalies positives et négatives viennent bousculer ce schéma simpliste, et les modalités de la métropolisation contribuent à expliquer ces anomalies : au coeur de l'Iran, le poids de la métropole téhéranaise vient interrompre la courbe descendante, tandis que celle-ci s'infléchit plus vite vers le bas si nous partons vers le nord-est, à travers la Transcausie puis l'Asie centrale ex-soviétiques : les effets de l'implosion du système communiste y sont sans doute aggravés par l'incapacité de métropoles rivales à dynamiser efficacement ces deux morceaux de l'empire déchu.

4 La question qui se pose est donc celle de la position de notre ensemble territorial dans le processus de métropolisation, comme le souligne Guy Burgel à propos du cas athénien : s'agit-il simplement d'une transition géographique dans l'espace, et d'un stade intermédiaire dans le temps, entre pays du Nord points de départ de la métropolisation et pays du Sud plus tardivement touchés, ou bien peut-on tenter de définir un modèle historique original donnant son caractère propre à notre aire d'étude ?

5 La démarche des géographes, majoritaires parmi les auteurs ayant contribué à ce dossier, suggère de procéder en deux temps correspondant à deux échelles emboittées : il faut tout d'abord, d'en-haut, en quelque sorte, identifier les métropoles en mesurant leur pouvoir d'attraction sur les territoires, puis descendre au niveau des métropoles ellesmême pour étudier les formes et les problèmes des espaces métropolisés.

Les métropoles, des villes de plus en plus attractives

6 Le terme même de métropole est né en Méditerranée orientale, dans le contexte de la colonisation des bassins méditerranéen et pontique à partir d'un certain nombre de cités grecques. La métropolis, la " cité-mère ", était liée par des rapports de domination à ses "filles" dispersées le long de côtes lointaines. L'Athènes antique peut ainsi apparaître comme la " métropole étymologique ", la "ville-mère " par excellence, à la fois centre d'une des plus puissantes thalassocraties de son époque et lieu de filiation de la cité démocratique, et Guy Burgel montre combien l'Athènes d'aujourd'hui doit à la mobilisation de cet héritage.

7 Ces liens, très linéaires, et rendus bien ténus par la précarité des communications, entre métropoles et colonies dans l'Antiquité hellénique peuvent être comparées avec les liens plus forts et plus diffus tissés autour d'elles par les métropoles contemporaines, qui font d'elles plus que de très grandes villes ou mégapoles ${ }^{8}$, et qu'il faut encore différencier en fonction de trois échelles emboîtées correspondant aux niveaux international, national et régional.

La domination des métropoles sur les territoires nationaux 
8 Première constatation, tous les pays de notre aire d'étude voient une agglomération dominer de loin la hiérarchie urbaine, tant par son poids démographique que par la richesse de ses fonctions. Autrement dit, l'échelle nationale est celle qui s'impose d'emblée, bien qu'elle constitue le niveau scalaire intermédiaire, et de fait, toutes ces «villes primatiales» sont la capitale politique d'un Etat, et d'un Etat fortement centralisé, au moins dans ses principes constitutionnels.

9 Toutes sauf une, Istanbul, mais c'est une exception qui confirme la règle, car il s'agit de l'ancienne capitale, dépossédée au profit d'Ankara quand la République de Turquie a succédé à l'Empire ottoman. Cette circonstance historique a créé en Turquie une situation - unique dans notre aire de référence mais pas exceptionnelle dans le monde, cf. les doublets Rome/Milan, Madrid/Barcelone, Casablanca/Rabat, Damas/Alep, Jérusalem/Tel Aviv etc... - de dyarchie urbaine. En effet, la nouvelle capitale Ankara a assumé toutes les fonctions de direction d'un Etat unitaire et centralisé : organes du gouvernement et Parlement, représentations diplomatiques étrangères, état-major de l'armée, sièges des nombreuses entreprises économiques d'Etat, institutions culturelles nationales, mais l'ancienne capitale a conservé sa primauté économique : premier port et première place de commerce du pays, premier centre industriel, première ville universitaire, elle rassemble la plupart des sièges sociaux des sociétés privées et l'essentiel du secteur privé culturel (édition, théâtre, écrivains et artistes).

10 Les deux villes se partagent ainsi la domination sur l'ensemble du pays, chacune exerçant son influence prépondérante dans un certain nombre de domaines, tout en se trouvant étroitement subordonnée à sa rivale dans d'autres domaines. Sur le plan politico-administratif, Istanbul n'est qu'un chef-lieu de département comme un autre même si le département d'Istanbul rassemble à lui seul $13 \%$ de la population du pays et dépend directement de la capitale au même titre que les petites villes de Hakkâri ou Tunceli dans l'Est montagneux. Inversement, sur le plan de l'activité commerciale, Ankara joue le rôle de centre de redistribution régionale de produits importés via Istanbul, et les établissements industriels ou tertiaires de la capitale dépendent en majorité de sièges sociaux implantés sur les rives du Bosphore.

11 Ce partage des fonctions de capitale entre les deux cités n'a pas suffi à les mettre sur un pied d'égalité, du fait du profond déséquilibre de départ entre une cité géante d'un demi-million d'habitants et une bourgade d'une vingtaine de milliers d'habitants. Certes une fois propulsée à la tête de la Turquie républicaine, Ankara a connu une croissance extrêmement rapide ${ }^{9}$, mais sans pouvoir jamais rattraper la capitale des sultans. Elle s'en est rapprochée au maximum en 1980, lors du recensement du 12 octobre, où sa population atteignait exactement les deux-tiers de la population stambouliote (1 877755 habitants contre 2772 708), mais Istanbul a repris son élan depuis, accentuant nettement son avantage avec 6620241 habitants au recensement d'octobre 1990 contre 2559471 pour la capitale politique. En ajoutant à l'agglomération d'Istanbul le reste du département et les trois départements limitrophes de Tekirdag, Kocaeli et Bursa, on arrive à 10317332 habitants, soit 18,3\% de la population de la Turquie, sur une superficie de $26600 \mathrm{~km}^{2}$, à peine supérieure à celle du seul département d'Ankara qui n'avait à la même date que 3236626 habitants sur 25700 $\mathrm{km}^{2}$. Voilà bien la métropolisation en marche !

12 Tous les autres Etats sont dominés par l'agglomération capitale, de façon plus ou moins nette. Deux cas sont à distinguer, celui des Etats anciennement constitués et celui des nouveaux Etats issus du démembrement de l'Union soviétique. Parmi les premiers, la 
Grèce montre la concentration la plus marquée : Athènes, avec 3,5 millions d'habitants, réunit le tiers de la population nationale, domine tous les secteurs d'activité et semble pomper toute la substance du pays, de telle façon que Guy Burgel peut se demander si elle n'est pas une "métropole exemplaire $»^{10}$. Téhéran, sans atteindre le même poids démographique relatif qu'Athènes - les 8 millions d'habitants du Grand Téhéran en 1991 représentaient quelque $12 \%$ de la population nationale - , domine tout autant la vie économique, sociale et politique du pays. Ici encore, le rôle de l'Etat dans le développement de sa jeune capitale a été considérable, et nous ne reviendrons pas dessus, ce sujet ayant été amplement débattu lors d'un colloque organisé en 1989 à Ivry par l'équipe de recherche "Sciences sociales du monde iranien contemporain " ${ }^{11}$. Eckart Ehlers a d'ailleurs montré que cette domination de la capitale n'était pas nouvelle : pour lui, «Persia's capital cities have in the past exerted a dominating grip over all parts of the country $»^{12}$. Beaucoup plus faiblement urbanisé, et épuisé par dixhuit ans de conflits, l'Afghanistan voit lui aussi sa capitale écraser la modeste armature urbaine du pays : Kaboul aurait de l'ordre de 1,5 millions d'habitants, soit 7\% seulement de la population totale, mais le tiers des citadins. Mais l'état du pays fait que nous sommes bien loin de la métropolisation!

Quant aux Etats de l'ex-empire soviétique, leur accession soudaine à l'indépendance a fait artificiellement monter d'un cran leurs capitales dans la hiérarchie urbaine. Naguère intégrées à des réseaux urbains couvrant l'URSS tout entière, ces villes se trouvent désormais propulsées au sommet des "pyramides urbaines » d'Etats petits ou moyens qu'elles dominent fortement. Leur poids relatif est le plus élevé dans les trois petits Etats de Transcaucasie, maximal dans le plus petit, l'Arménie, dont la superficie $\left(29800 \mathrm{~km}^{2}\right)$ est inférieure à celle du plus grand département turc (Konya, $38257 \mathrm{~km}^{2}$ ). Sa capitale Erevan réunit le tiers des habitants de la république (1,3 sur 3,8 millions d'habitants ${ }^{13}$ ), alors que Tbilissi et Bakou (au sens du Grand Bakou) concentrent respectivement le quart des Géorgiens et des Azerbaïdjanais. Jean Radvanyi, en étudiant leur position respective, souligne l'importance du fait national, qui différencie profondément ces trois pays et leur capitale respective, situation qu'on pourrait comparer à celle des pays baltes.

Les nouvelles républiques d'Asie centrale sont plus étendues, moins densément peuplées, et présentent plus de ressemblances entre elles du fait de leur appartenance commune au monde turco-iranien, ce qui ne suffit pas à éviter rivalités et conflits. Les cinq capitales y occupent partout un premier rang incontesté, avec des poids démographiques et économiques très contrastés, depuis Tachkent, la «ville de pierre » multimillionnaire, jusqu'à Douchanbé, le «marché du lundi» d'allure encore très provinciale.

L'émergence de métropoles régionales

Dans une partie de ces pays, une ou plusieurs villes «de province", le plus souvent localisées à la périphérie du territoire, ont cependant atteint un développement et une force d'attraction suffisants pour manifester une certaine tendance à l'autonomie vis-àvis de la capitale. Elles présentent une gamme complète de fonctions de niveau régional, et se sont constitué une zone d'influence bien caractérisée. On peut s'interroger sur le bien fondé de la dénomination de « métropole » dans ce cas. Elle fait clairement référence au concept de métropoles d'équilibre proposé dans la France des années 1960 et progressivement entré dans les faits en résultat des politiques d'aménagement du territoire. La déficience de ces dernières dans les pays qui nous 
intéressent doit nous inciter à la prudence dans la transposition de cette appellation, qui ne peut être tentée que pour un petit nombre de villes, dans des Etats assez étendus et différenciés pour que ce niveau intermédiaire des métropoles régionales vienne s'intercaler entre la capitale et des centres régionaux plus modestes.

Il n'est donc pas question de prétendre en identifier dans les petits Etats transcaucasiens, qui ne dépassent guère la dimension d'une région de bien d'autres pays, et dont les capitales ne laissent pas de place à un développement urbain autonome. De même la faible population totale, le taux d'urbanisation modeste et l'existence de vastes espaces désertiques ou montagneux vacants excluent de rechercher des métropoles régionales au Turkménistan, au Tadjikistan et au Kirghizistan. La question peut en revanche se poser dans l'immense Kazakhstan, où les deux principales aires de peuplement sont disjointes, piedmont des Tian Chan à population kazakh au sud-est et bande pionnière agricole et industrielle à majorité russophone au nord. La capitale Almaty est très décentrée au coeur de la première de ces deux aires, ce qui laisse la possibilité de s'affirmer aux principales villes de la seconde, Sémipalatinsk ou Karaganda. En Ouzbékistan de même, bien que l'aire de peuplement y soit plus restreinte et plus continue, la position excentrique de Tachkent permet de s'interroger sur le degré d'autonomie de Samarkand, qui bénéficie de plus de son prestige de cité historique multiséculaire.

17 Ce rôle de l'histoire se retrouve dans les Etats méridionaux aux réseaux urbains plus développés. Ainsi la re-création de la Grèce moderne en deux étapes bien distinctes a-telle certainement contribué à l'affirmation de Salonique comme métropole de la " Nouvelle Grèce » du nord... tout en lui donnant sur Athènes un retard qui semble impossible à rattraper. Georges Prévélakis s'attache à montrer les changements successifs de valeur d'une situation de toutes façons remarquable, au débouché du couloir ouvrant la voie des Balkans, à laquelle Guy Burgel oppose le «site sans situation" d'Athènes "placée au centre des régions les plus déprimées démographiquement et économiquement de l'Hellade contemporaine ", et les efforts des Saloniquiotes pour secouer la tutelle athénienne et sortir du provincialisme dans lequel les dirigeants nationaux ont enfermé leur cité.

Turquie, qui bénéficie du réseau urbain le mieux développé et le plus différencié de notre aire d'étude comme de tout le Proche-Orient ${ }^{14}$, trois métropoles régionales ont $\mathrm{pu}$ s'affirmer en plus des deux capitales, l'ancienne et la nouvelle, dans la moitié occidentale du pays. Toutes trois tiennent leur rôle de leur importance commerciale et industrielle acquise au cours des dernières décennies de l'Empire ottoman : Izmir qui a animé le développement des cultures commerciales dans l'ensemble des vallées de la façade égéenne, drainées vers son port par un éventail de voies ferrées ${ }^{15}$, Bursa, cité historique et station thermale qui a développé à la manière lyonnaise tout un complexe industriel à partir du travail de la soie ${ }^{16}$, et Adana, ville-atelier qui a transformé dans ses usines la production cotonnière de la plaine de la Çukurova ${ }^{17}$. Toutes trois dépassent aujourd'hui le million d'habitants et contribuent à étendre les effets de la métropolisation à une grande partie de la Turquie de l'Ouest développée, industrialisée et urbanisée, qu'un " gradient de développement ${ }^{18}{ }_{\text {» }}$ très net oppose à la Turquie de l'Est en retard.

19 En Iran de même quatre agglomérations millionnaires, Tabriz, Machhad, Esfahân et Chirâz, esquissent un contrepoids à l'influence téhéranaise. Les racines historiques de leur rôle de métropole régionale sont plus anciennes qu'en Turquie, puisque toutes 
quatre ont exercé avant Téhéran, pendant un temps plus ou moins long, la fonction de capitale politique de la Perse, au gré de la succession des dynasties : Tabriz a été le centre des principautés Kara Koyounlou puis Ak Koyounlou au XVe siècle, puis la première capitale des Safavides avant que les guerres contre les Ottomans ne leur fissent déplacer leur siège vers des positions moins exposées, Qazvin sous Châh Tahmâsp I (1524-1576), puis Esfahân en 1598 sous Châh 'Abbâs I. Cette dernière a été la capitale la plus durable (cinq-quarts de siècle jusqu'à l'invasion afghane de 1722) et la plus prestigieuse de la Perse moderne, atteignant le demi-million d'habitants lorsque le voyageur français Chardin ${ }^{19}$ la visita en 1673 . La période troublée du XVIIIe siècle vit la capitale beaucoup plus brièvement installée par Nâder Châh Afchar à Machhad, qui doit finalement plus son rayonnement à son rôle de métropole religieuse des chiites iraniens lié au pèlerinage sur la tombe de l'Imam Rezâ, puis par le Régent Karim Khân-e Zand à Chirâz, avant que la nouvelle dynastie des Qâdjârs ne se fixe à Téhéran. Une étude reste à réaliser sur l'attraction de ces quatre grandes villes, qui surpassent de loin les autres chefs-lieux de province.

De même en Afghanistan, Etat tardivement constitué ${ }^{20}$, deux autres villes, Hérat au nord-ouest et Kandahar au sud, jalonnaient avec Kaboul à l'est le « croissant pachtoun » qui entoure par le sud le massif de l'Hindou Kouch. Toutes trois avaient été en leur temps le centre d'entités politiques indépendantes et conservaient une importance comparable lorsque le premier Etat afghan s'est constitué au XVIIIe siècle. Le chef de la confédération pachtoune des Dourrrâni, Ahmad Châh, couronné roi en 1747, choisit d'abord Kandahar pour capitale puis son fils Timour transféra sa capitale à Kaboul, avant que le royaume ne se désintègre en trois principautés centrées sur les trois villes. C'est seulement lors de la réunification de l'Afghanistan au cours de la seconde moitié du XIXe siècle en tant qu'Etat-tampon maintenu entre les Empires russe et britannique que Kaboul a définitivement assis sa fonction de capitale, toujours menacée par les tendances centrifuges. De plus Kandahar et surtout Hérat ont connu de profondes destructions au cours des guerres, contre l'occupant soviétique puis entre factions rivales, qui ensanglantent le pays depuis dix-huit ans, et semblent avoir perdu pour quelque temps leur capacité traditionnelle à contrebalancer l'influence de Kaboul.

21 La question finale qui se pose à propos de ces métropoles régionales en émergence est de savoir si elles affaiblissent le rayonnement des agglomérations primatiales ou si au contraire elles relaient la capitale pour diffuser vers d'autres parties du pays le processus de métropolisation. Par ailleurs, on pourrait soutenir le paradoxe que les premières métropoles régionales sont les capitales, au moins dans les cas d'Istanbul et de Téhéran qui ont développé autour d'elles des liens très denses avec une « région métropolitaine " clairement emboîtée dans leur aire d'influence nationale étendue à tout le pays ${ }^{21}$.

A la recherche d'une affirmation internationale

Pendant que s'affirment en-dessous d'elles ces métropoles régionales, les capitales regardent vers le haut, autrement dit cherchent à développer leurs fonctions de niveau international, en se tournant dans des directions différentes et en obtenant des résultats inégaux.

Cette dimension internationale est fondamentale dans le cas d'Athènes, dont Guy Burgel analyse les traits de " métropole mondiale à l'heure européenne ». Deux raisons à cela : tout d'abord, l'hellénisme dont Athènes est la métropole déborde largement des frontières de la petite Grèce. Si les tentatives d'expansion territoriale en continu ont 
échoué, la «Grande Idée » du rattachement de la façade égéenne de l'Asie Mineure, l'Ionie antique, ayant dû être abandonnée en 1922-23 à l'issue de la guerre grécoturque, et l'intervention brutale de la Turquie en 1974 (dite "opération Attila ») ayant coupé court à l'Enôsis, l'annexion de Chypre à la Grèce, et instauré la partition durable de l'île, Athènes et l'Etat grec sont au centre d'une diaspora disséminée sur des espaces très étendus et très diversifiée, avec laquelle ils entretiennent des relations ambiguës et changeantes ${ }^{22}$. D'autre part les activités maitresses de l'économie grecque, le tourisme international et la marine marchande, sont l'une et l'autre par essence tournées vers l'espace mondial, et ont constitué, comme le remarque Guy Burgel, "des apprentissages féconds pour les ouvertures contemporaines » de la mondialisation, et aussi de l'intégration européenne.

Istanbul et Téhéran ne manquent pas d'ambitions internationales, mais éprouvent plus de difficultés à les concrétiser. En partie rivales, elles regardent plutôt vers des espaces différents. Istanbul, seule ville du monde à cheval sur deux continents, met volontiers en avant la symbolique de ses deux ponts sur le Bosphore reliant l'Europe à l'Asie et illustre la position de la Turquie dans son ensemble à l'intersection de plusieurs espaces $^{23}$. Elle est d'une certaine façon l'instrument de l'ouverture de la Turquie vers l'Europe de l'Ouest où vivent plus de 2 millions de Turcs émigrés comme vers la « deuxième Europe » des Balkans et de la mer Noire. Elle tient également sa part dans les relations de la Turquie avec ses deux autres aires d'influence, le Moyen-Orient et les pays musulmans du Caucase et de l'Asie centrale, mais tout l'aspect politique de ces relations est bien évidemment maîtrisé par Ankara. C'est donc principalement dans les champs économique et culturel que s'exprime l'internationalisation des fonctions d'Istanbul, analysée récemment par Jean-François Pérouse ${ }^{24}$.

Téhéran de son côté associe dans son rayonnement international une dimension idéologique, en tant que centre de la première révolution islamiste, et une dimension beaucoup plus pragmatique. La première a quelque difficulté à s'affirmer car les musulmans sunnites, même les plus fondamentalistes, restent méfiants vis-à-vis de la capitale des révolutionnaires chiites, et si les liens entre Téhéran et le Hezbollah chiite libanais sont bien connus, les relations evec les chiites arabes dans leur ensemble sont malgré tout freinées par un sentiment nationaliste opposant Arabes et Iraniens. Si la dimension religieuse n'est évidemment pas absente des efforts considérables des Iraniens pour pénétrer dans les nouvelles républiques d'Asie centrale, elle passe au second plan derrière les préoccupations culturelles et économiques. Malgré l'existence d'une longue frontière commune avec le Turkménistan, les relations régionales transfrontalières semblent s'être assez peu développées, et c'est bien Téhéran qui constitue le passage obligé pour la plupart de ces échanges.

26 La notion de relations internationales est plus complexe du côté nord de notre aire d'étude, car les Etats issus de l'URSS ont vu accéder au statut international des relations préexistantes entre eux ou avec la Russie. L'instauration de ces frontières supplémentaires, dans un contexte politique souvent tendu, quand il n'est pas allé jusqu'à l'affrontement militaire, a plutôt imposé un frein à ces relations.

27 Daniel Balland le montre éloquemment dans le cas de l'Asie centrale, où Tachkent avait développé dans le cadre de l'Union soviétique de nombreuses fonctions desservant le territoire de l'Asie centrale tout entière. Celle-ci a souvent constitué dans le système soviétique une " région économique » unique, ainsi pendant les $4 \mathrm{e}, 5 \mathrm{e}$ et $6 \mathrm{e}$ plans entre 1946 et 1960, ou bien associait le Kazakhstan et une «Asie moyenne» (Srednaia Asia) 
réunissant les quatre autres républiques, comme dans le découpage en vigueur en 1989 juste avant l'écroulement de l'URSS ${ }^{25}$. Les anciennes républiques fédérées devenues indépendantes se sont trouvées beaucoup plus réticentes pour dépendre d'une capitale désormais « étrangère » pour des services de haut niveau et ont tenté de s'affranchir de ce qui était perçu comme une tutelle de Tachkent. Les perspectives d'intégration économique et politique, qui ne pourraient que profiter à la capitale de l'Ouzbékistan, paraissent bien ténues à l'heure actuelle.

28 A fortiori, les trois petits Etats transcaucasiens, qui ne vivaient déjà pas en bonne entente dans la fédération, bien qu'ils fussent rangés - par une décision d'en-haut dans la même région économique, n'ont eu de cesse de se déchirer une fois l'indépendance acquise. Du coup l'écheveau des relations entre leurs trois capitales, que Jean Radvanyi tente de démêler, est plus fait de rivalités que de complémentarités. Les frontières quasiment étanches font obstacle à tout échange, et chacune de ces villes tente de s'affirmer contre les deux autres et établit séparément des liens avec les pays voisins (Tbilissi et Bakou avec la Turquie, Bakou mais aussi Erevan avec l'Azerbaidjan iranien) comme avec des partenaires lointains.

Structure et organisation des espaces métropolises

En passant de l'échelle des territoires nationaux à celle des métropoles elles-mêmes, nous constatons que leur grande dimension, si elle est une nécessité pour avoir un poids crédible vis-à-vis de l'extérieur, n'est pas sans poser des problèmes variés, évoqués par une bonne partie des contributions qui suivent. Leur extension spatiale oblige à repenser les structures politico-administratives, dont les responsables seront confrontés à de difficiles problèmes de gestion urbaine.

Une nouvelle morphologie de l'espace

Les métropoles sont des villes de plus en plus étendues. La croissance démographique et économique de ces grandes agglomérations entraîne une importante consommation d'espace. L'espace urbanisé en continuité couvre ainsi à Istanbul un triangle de plus de $60 \mathrm{~km}$ de base (le front de mer de la Marmara) et de quelque $30 \mathrm{~km}$ de hauteur (l'axe du Bosphore). Téhéran, à partir du quadrilatère de $5 \mathrm{~km}$ de côté urbanisé vers 1950 , s'est étalée sur $25 \mathrm{~km}$ dans le sens nord-sud, perpendiculairement au piedmont, de Chemirân sur les premières pentes de l'Alborz à Rey tout en bas, et sur une quarantaine de $\mathrm{km}$ dans le sens ouest-est, le long du piedmont. Athènes a entièrement rempli la petite plaine de l'Attique et va bien au-delà etc...

31 A la notion de superficie bâtie, ou plus exactement urbanisée, il convient d'ajouter celles de continuité et de densité. Dans chacun des trois cas mentionnés ci-dessus, l'aire d'urbanisation continue que nous venons de délimiter grossièrement est entourée d'une couronne externe de plus faible densité globale et très hétérogène. Cette couronne s'éloigne notablement de la forme circulaire du modèle théorique en auréoles. A Téhéran, elle s'est principalement développée vers l'ouest, où Bernard Hourcade nous montre l'émergence d'une véritable banlieue, associant des agglomérations constituées autour de noyaux préexistants, telles Karadj et Varâmin, et d'autres nées de rien, telles Eslâm-Shahr ou Rejâ'i-Shahr, entrecoupées de terroirs cultivés et de morceaux de steppe vacants. Cette urbanisation périphérique est plus diffuse à Athènes et Istanbul. Le Grand Bakou, de même, juxtapose la ville dense, d'un seul tenant en arrière de la cité musulmane traditionnelle serrée dans ses murs, et le semis de petites villes égaillées à travers toute la péninsule d'Apchéron. 
32 Mais cette croissance ne doit pas être vue en termes purement quantitatifs de surfaces urbanisées et de densités : les modalités de l'urbanisation et les formes urbaines résultantes doivent également être prises en compte. Stéphane Yerasimos insiste avec raison sur la nécessité de décrire les processus de production de l'espace urbain, qui associent dans le cas d'Istanbul la reproduction de l'espace urbain légal dans les aires déjà urbanisées, la production illégale d'espace urbain, devenue la principale forme d'extension urbaine, la légalisation de cet espace urbain illégal, et les opérations groupées portant sur des emplacements isolés à la périphérie. De même à Athènes la construction associe-t-elle depuis l'après-guerre les polykatoikies, immeubles d'appartements banals, et les constructions illicites des banlieues pauvres, double modèle qui s'est diffusé dans l'ensemble du pays, entre autres à Salonique où il ne reste presque plus rien du centre ancien à cachet ottoman.

33 La structure urbaine ne se limite pas à la différenciation de l'habitat, elle se définit également par la répartition des fonctions, le zonage, volontariste ou de fait. Les fonctions tertiaires de haut niveau sont plus ou moins étroitement concentrées, mais dans tous les cas l'étalement métropolitain conduit à une certaine déconcentration, voire à une structure franchement polycentrique. A Athènes, la concentration était extrême, rassemblant dans un "triangle d'or " très restreint le pouvoir politique, la puissance économique, l'influence intellectuelle et la richesse du commerce de détail. Mais le transfert vers l'extérieur du marché de gros et de nombreux bureaux et la création de nouveaux centres commerciaux sont venus démultiplier la centralité athénienne. La métropole stambouliote, elle, était polycentrique dès le départ, en fait dès le Moyen-Age avec la ville des marchands européens, Galata, faisant face du côté opposé de la Corne d'Or à Constantinople devenue Istanbul. A partir de là, les centres secondaires se sont multipliés depuis une vingtaine d'années, sous plusieurs formes : artères rassemblant les commerces de luxe (Harbiye du côté européen ou l'avenue de Bagdad du côté asiatique), nouveaux centres d'affaires desservis par les rocades (Levent, Maslak), plateforme logistique extérieure (Ikitelli). Téhéran aussi est caractérisée par la structure bipolaire de son centre, modélisée par Martin Seger ${ }^{26}$. Elle présente une forte dissymétrie, la dynamique d'ensemble tirant vers le haut le centre de gravité du commerce, malgré la résistance inattendue du bazar, attestée par la thèse récente de V. Shâli Amini ${ }^{27}$ qui a comparé la situation de 1991 à l'inventaire effectué par M. Seger en 1973 et repéré plusieurs cas d'avancée aux dépens de l'habitat. Là encore, l'éclatement de l'agglomération vers des banlieues très éloignées oblige à la démultiplication des structures commerciales, mais ces banlieues ne semblent pas avoir accueilli de fonctions directionnelles déconcentrées.

Une autre activité dont la présence est généralement assez discrète dans les métropoles de l'Occident développé, à moins qu'elle ne soit rejetée en périphérie, est l'industrie. Or un trait original des métropoles présentées ici est le poids relatif important de l'industrie. Toutes sont, et de loin, le premier centre industriel du pays qu'elles dominent, et les activités industrielles s'entremêlent de façon complexe avec les autres utilisations du sol urbain, suivant des logiques que Harald Standl a étudiées en détail dans le cas d'Istanbul ${ }^{28}$. Les résultats ne sont pas toujours très heureux en matière de respect de l'environnement, témoin le nuage de pollution qui couvre d'ordinaire Athènes, ou la "Ville Noire" (Qara Chèhèr) datant de la première génération d'exploitation du pétrole à Bakou et méritant parfaitement son nom. De cette présence physique de l'industrie découle également sa présence sociale : à Istanbul, Kemal 
Karpat ${ }^{29}$ a montré comment les gecekondu (les quartiers spontanés, voir les articles de $\mathrm{S}$. Yerasimos et J-F. Pérouse), au moins ceux de la première génération, étaient localisés en fonction de l'accessibilité aux zones industrielles.

Il est particulièrement intéressant d'aller voir la métropole sur ses marges, sur le front du processus d'urbanisation. C'est ce qu'ont fait Jean-François Pérouse à Istanbul et Nouchine Yavari d'Hellencourt à Téhéran en se rendant respectivement à Gaziosmanpasa, au nord-ouest de la partie européenne d'Istanbul, et à Eslâm-Shahr, à une vingtaine de kilomètres au sud-ouest de Téhéran. Ces études à grande échelle présentent le grand avantage de pouvoir aborder de façon concrète la réalité sociale de la métropole, car les échantillons urbains ainsi prélevés sont autant d'échantillons de groupes sociaux, et on entre de plain-pied dans la différenciation sociale de l'espace, en voyant par exemple comment, à Eslâm-Shahr, les petites rues résidentielles constituent un espace communautaire, d'utilisation changeante au cours de la journée, qui marque la transition entre l'espace privatif des maisons et l'espace étranger et ambigu des grandes artères commerçantes.

L'organisation politico-administrative de l'espace métropolitain

Par leur dimension et leur complexité, les métropoles cadrent mal avec les découpages administratifs habituels. Elles ont souvent débordé de leur périmètre d'origine sur des banlieues de statut administratif différent, mais qui partagent avec elles de nombreux problèmes exigeant des réponses communes. L'organisation métropolitaine est donc un défi lancé aux responsables politiques et aux juristes. L'aire d'étude recèle une grande variété de situations.

(a) fasure possible, l'absence de toute entité administrative commune est illustré par Athènes : l'agglomération est émiettée entre plus de cinquante communes, la région urbaine est répartie entre plusieurs départements, et il n'y a même aucune unité statistique regroupant systématiquement les données concernant Athènes. Guy Burgel souligne à ce propos le paradoxe de la décentralisation, qui, en prétendant doter de pouvoirs accrus des communes trop petites et dépourvues de moyens financiers, ramène en fait dans les mains de l'Etat la capacité de choix.

Téhéran semblait mieux pourvue au départ, car sa croissance s'est effectuée jusqu'à la fin des années 1970 dans les limites d'un périmètre municipal étendu, enfermant les contours de l'aire effectivement urbanisée dans un quadrilatère de $25 \times 20 \mathrm{~km}$, subdivisé en 20 arrondissements ${ }^{30}$. Hors de ces limites, seule la ville-satellite de Karadj, $40 \mathrm{~km}$ plus à l'ouest, ayant le statut de préfecture du département voisin, s'accroissait notablement. Or l'apparition de la banlieue, pour l'essentiel consécutive à la révolution islamique comme le montre Bernard Hourcade, a juxtaposé à la capitale des agglomérations dépassant les 100000 habitants, mais n'ayant au départ pratiquement aucune existence légale, d'autant qu'elles ne s'appuyaient même pas sur un noyau villageois ancien. La reconnaissance institutionnelle est venue après coup, longtemps après, puisqu'Eslâm-Shahr, la plus anciennement développée et la plus connue de ces agglomérations, n'est devenue qu'en 1996 chef-lieu d'un nouveau département. Si l'expression de Grand Téhéran est apparue à plusieurs reprises dans les documents officiels, elle ne désigne qu'un périmètre variable utilisé pour des études de planification successives, mais ne s'est jamais concrétisée dans une entité administrative ni même statistique.

39 La Turquie, au contraire, a pris conscience du décalage entre structures administratives et réalité urbaine et a cherché à y remédier en adoptant en 1984 une loi instituant des 
municipalités métropolitaines (Büyük Sehir Belediyesi, littéralement «municipalité de grande ville»), dont Yeseren Eliçin-Arikan analyse les clauses et les effets dans ce volume, en reprenant les apports de sa thèse ${ }^{31}$. La loi crée pour les plus grandes villes, au-dessus d'un seuil de 300000 habitants, un système à deux niveaux : une municipalité métropolitaine et un nombre plus ou moins élevé de municipalités d'arrondissement (ilçe belediyesi), ou plus exactement à trois niveaux puisque les municipalités d'arrondissement sont subdivisées, tout comme les municipalités ordinaires, en muhtarlik ou quartiers, unités de proximité où un muhtar, élu comme les conseillers municipaux et maires des deux niveaux supérieurs, assure diverses tâches de gestion quotidienne (état-civil, recensement des recrues, relations des citoyens avec les autorités etc...). L'articulation des pouvoirs et des compétences entre les deux niveaux de municipalités conditionne l'aménagement et le fonctionnement des grandes agglomérations. On notera au passage que le seuil démographique établi par la loi est assez bas et va bien au-delà des cinq villes (les deux capitales Istanbul et Ankara et les trois métropoles régionales Izmir, Adana et Bursa) auxquelles nous avons reconnu des fonctions métropolitaines. En fait, la loi a été mise en application progressivement, en commençant par les trois plus grandes villes, et en 1990 huit villes seulement sur les 17 ayant vocation à accéder à ce statut étaient effectivement divisées en arrondissements : les cinq villes précitées plus Gaziantep, Konya et Kayseri.

Un système comparable semblait exister en Azerbaïdjan à la fin de l'ère soviétique, à en juger par une planche de l'Atlas de l'Azerbaïdjan ${ }^{32}$ représentant le «Soviet urbain de Bakou » : il couvrait alors la plus grande partie de la péninsule d'Apchéron (moins la ville de Soumgaït au nord-ouest) et une étroite bande littorale étirée sur plus de $80 \mathrm{~km}$ vers le sud. Il était subdivisé en dix arrondissements (rayon), dont cinq arrondissements urbains se partageant la ville même de Bakou, ceux d'Octobre, des 26 Commissaires de Bakou, de Nessimi, Narimanov et Chaumyan, et cinq arrondissements périurbains. Plusieurs questions demandent un complément d'information : quelles étaient les attributions respectives des deux niveaux ? la même organisation administrative étaitelle appliquée dans les autres républiques fédérées ? et surtout a-t-elle survécu à la chute du régime communiste?

De multiples problèmes d'aménagement

41 L'organisation de ces pouvoirs municipaux et locaux est essentielle pour assurer la gestion de ces métropoles confrontées à des problèmes variés affectant la vie quotidienne des habitants, et dont l'accumulation pose un problème théorique évoqué plus loin par Jacques Lévy : au-delà d'un certain seuil (comment le chiffrer ?), les inconvénients liés à la congestion urbaine l'emporteraient-ils sur les avantages de la proximité et de la richesse des fonctions? Autrement dit, le rendement de la «machine urbaine" commencerait-il alors à décliner ? Il est difficile de se prononcer, nous devons nous contenter de constater que les métropoles envisagées ici donnent un bon échantillon de ces problèmes métropolitains.

Il s'agit d'abord de faire face à la croissance démographique de ces agglomérations en assurant à tous un logement décent et l'accès à des équipements de proximité. Or le coup d'oeil jeté précédemment sur les modes d'urbanisation montre qu'on est souvent loin du compte, et que seule la pratique des constructions illégales a permis de satisfaire les besoins en quantité, sinon toujours en qualité. Ce secteur informel du bâtiment joue un rôle décisif aussi bien à Athènes qu'à Istanbul, à Ankara qu'à Téhéran, 
à Salonique que dans les métropoles régionales turques, et il serait bien étonnant qu'il soit totalement absent des capitales post-communistes.

La circulation et les transports urbains sont un autre casse-tête pour les édiles. Les métropoles sont aussi des espaces surmotorisés par rapport au reste de chaque pays, et ce suréquipement relatif aboutit vite à la congestion de la circulation et à des pics de pollution atmosphérique. Il est d'ailleurs symptomatique que deux types de mesures radicales à l'encontre des véhicules individuels aient été inaugurées dans deux des métropoles de la région : la circulation alternée suivant les numéros pairs ou impairs des plaques d'immatriculation à Athènes et l'interdiction du centre-ville (sur $18 \mathrm{~km}^{2}$ environ) aux voitures individuelles une grande partie de la journée à Téhéran. Dans les deux cas on attend fébrilement un métro projeté depuis longtemps mais dont la (coûteuse) réalisation semble piétiner. Il viendrait compléter une gamme variée de modes de transports en commun : autobus, minibus, taxis individuels ou collectifs qu'il convient de coordonner. La gamme est encore plus complète à Istanbul, qui a d'autre part construit deux rocades autoroutières menant aux deux ponts sur le Bosphore : autobus municipaux et privés (desservant les mêmes lignes en complément des premiers vu la pénurie de véhicules), minibus et dolmus (taxis collectifs), tramways, à l'ancienne le long d'Istiklâl Caddesi et modernisés dans la ville historique, sans oublier les bateaux urbains, vapur (vaporetti à grande capacité) et deniz otobüsü (" autobus de mer », en fait vedettes rapides pour des liaisons express), en attendant le métro dont le chantier avance. Bakou, elle, dispose d'un métro depuis les années 1970, mais partage avec ses voisines de Transcaucasie, et sans doute d'Asie centrale, le mauvais état de la voirie et de nombreux équipements.

Ces fortes concentrations de population posent enfin des problèmes d'approvisionnement et d'évacuation - ou plus globalement de gestion de l'écosystème urbain - : approvisionnement en denrées alimentaires, en eau et en énergie, évacuation des eaux usées et des déchets domestiques et industriels. Tout ceci, une fois encore, suppose une coordination à l'échelle métropolitaine, qui est inégalement assurée. Des accidents graves, comme l'explosion en 1994 d'une décharge précipitant un torrent de boue sur des gecekondu à Ümraniye, sur la rive asiatique du Bosphore, ont contribué à alerter l'opinion sur la nécessité d'une gestion plus écologique de la ville, mais il reste énormément à faire dans ce domaine.

Par leur croissance démographique et spatiale, par leur poids accru dans le fonctionnement économique des Etats, par leurs difficultés à organiser la maîtrise de leur espace et la gestion des besoins des habitants, les métropoles de l'aire d'étude des CEMOTI, d'Athènes à Tachkent, sont bien représentatives du mouvement contemporain de métropolisation, tout en présentant bien des nuances dont rendront compte les articles qui suivent. 


\section{NOTES}

1. Une des meilleures présentations des différentes facettes de ce phénomène est donnée dans La Mondialisation au-delà des mythes, Paris, La Découverte (Les Dossiers de l'état du monde), 1997, $174 \mathrm{p}$.

2. Voir entre autres J-P. LERESCHE, D. JOYE et M. BASSAND, Métropolisations, Genève, Georg, 1995.

3. Pierre VELTZ, Mondialisation, villes et territoires. L'économie d'archipel, Paris, P.U.F., 1996.

4. Saskia SASSEN, The global City: New York, London, Tokyo, Princeton, Princeton University Press, 1991, 397 p.

5. François ASCHER, Métapolis ou l'avenir des villes, Paris, Odile Jacob, 1995, 347 p.

6. Jacques LEVY, «Six idées sur la métropolisation partagée », in Jean-Claude NEMERY

(dir.), Le renouveau de l'aménagement du territoire en France et en Europe, Paris, Economica/

DATAR/Univ. de Reims Champagne-Ardenne, 1994, pp. 255-264.

7. Jean DOMINGO et Bernard ELISSALDE, L'espace mondial depuis les années 30, une géographie changeante, Rosny, Bréal, 1994, 220 p. (III, ch. 4, pp. 189-206).

8. Ce qui n'empêche pas d'appliquer également ce dernier terme à une partie au moins des agglomérations qui nous intéressent ici, témoin l'article de Stéphane YERASIMOS, "Istanbul : la naissance d'une mégapole », Revue Géographique de l'Est, 1997/2-3, pp. 189-215.

9. Analysée par Jean-François PEROUSE dans sa thèse D'Angora à Ankara (1919-1950) : la naissance d'une capitale, Thèse nouveau régime, Université de Reims ChampagneArdenne, 1994, 642 p. et sur une période plus longue dans son article « Ankara : la croissance équivoque d'une création-symbole ", Revue Géographique de l'Est, 1997/2-3, pp. 217-242.

10. Voir également Georges PREVELAKIS, Athènes : naissance et renaissance d'une ville méditerranéenne, Document de synthèse pour l'habilitation à diriger les recherches, Université de Paris-Sorbonne, 1996, 219 p.

11. Chahryar ADLE et Bernard HOURCADE (dir.), Téhéran capitale bicentenaire, ParisTéhéran, Institut Français de Recherche en Iran, 1992, 386 p.

12. Eckart EHLERS, « Capitals and spatial organization in Iran : Esfahan, Shiraz, Tehran », id., pp. 155-172 (passage cité p. 171).

13. Ces chiffres et les suivants proviennent des Images Economiques du Monde 1998, Paris, SEDES, 1997.

14. Marcel BAZIN, « Le réseau urbain de la Turquie », Travaux de l'Institut de Géographie de Reims, $\mathrm{n}^{\circ} 65-66,1986$, pp. 89-113,

et, pour une comparaison avec les pays voisins du Proche-Orient, Marcel BAZIN, « Urbanisation et systèmes urbains au Proche-Orient », Revue Géographique de l'Est, 1997/2-3, pp. 115-139.

15. Ahmet Necdet SÖZER, "Izmir, métropole de l'Egée", Travaux de l'Institut de Géographie de Reims, $\mathrm{n}^{\circ}$ 65-66, 1986, pp. 115-131.

16. Reinhard STEWIG, Bursa, Nordwestanatolien. Auswirkungen der Industrialisierung auf die Bevolkerungs- und Sozialstruktur einer Industriegrossstadt im Orient, Teil 1, 1980, et Teil 2, 1986, Kiel, Kieler Geographische Schriften, H. 51 et 65.

17. Lothar ROTHER, Die Städte der Çukurova : Adana - Mersin - Tarsus, Tübingen, Tübinger Geographische Studien, 42, 1971. 
18. West-Ost Gefälle suivant l'expression de Wolf-Dieter HÜTTEROTH, Türkei, Darmstadt, Wissentschaftliche Buchgesellschaft, 1982.

19. Jean CHARDIN, Voyage de Paris à Ispahan, Paris, La Découverte / Maspero, 1983, 2 vol. (coll. La Découverte, 64 et 65).

20. Voir les modalités de sa genèse dans le chapitre VIII « L'Afghanistan ou l'antination » de Xavier de PLANHOL, Les Nations du Prophète. Manuel géographique de politique musulmane, Paris, Fayard, 1993, pp. 593-678.

21. Voir Marcel BAZIN, « Les fonctions métropolitaines d'Istanbul, du Caire et de Téhéran, réflexions comparatives ", communication au colloque Métropoles, organisé à Istanbul par le Département francophone de Sciences Politiques et Administratives de l'Université de la Marmara, 23-25 septembre 1992 (en particulier les fig. 2 et 3).

22. Analysées par Georges PREVELAKIS dans son article « Les espaces de la diaspora hellénique et le territoire de l'Etat grec », in G. PREVELAKIS (dir.), Les réseaux des diasporas / The networks of diasporas, Nicosie, KYKEM, 1996, pp. 53-68.

23. Voir les actes du colloque tenu à Montpellier par le GDR 832 du CNRS sur cette question en septembre 1995 : Marcel BAZIN, Salgur KANÇAL et Jacques THOBIE (dir.), La Turquie entre trois mondes, à paraître aux éditions L'Harmattan, et une brève chronique : Marcel BAZIN, « Turquie. Entre Europe et Asie », in Le Livre de l'année 1997, Paris, Larousse, 1997, pp. 437-439.

24. Jean-François PEROUSE, «L'internationalisation des fonctions d'Istanbul », communication au colloque international Méditerranée orientale et mer Noire entre mondialisation et régionalisation organisé en septembre 1997 à Antalya par le GDR 832 du CNRS et l'Université de la Méditerranée.

25. Jean RADVANYI, L'URSS : Régions et Nations, Paris, Masson, 1990, pp. 13-14 et fig. 3 et 6.

26. Martin SEGER, « Segregation of retail facilities and the bipolar city center of Tehran ", in Ch. ADLE \& B. HOURCADE, op. cit.

27. Vahid SHALI AMINI, Bazar de Téhéran, Thèse de doctorat n. r., Université de ParisSorbonne, 1996, $472 \mathrm{p}$.

28. Harald STANDL, Der Industrieraum Istanbul. Genese der Standortstrukturen und aktuelle Standortprobleme des verarbeitenden Gewerbes in der türkischen Wirtschaftsmetropole, Bamberg, Fach Geographie an der Universität, 1994 (Bamberger Geographische Schriften, H. 14), 177 p. + annexes.

29. Kemal H. KARPAT, The Gecekondu : Rural Migration and Urbanization, Cambridge, Cambridge University Press, 1976.

30. Voir le découpage de ces arrondissements ainsi que divers aspects de leur différenciation socio-spatiale dans Masserat AMIR-EBRAHIMI, « L'image sociogéographique de Téhéran en 1986 », in Ch. ADLE \& B. HOURCADE, op. cit., pp. 267-279. 31. Yeseren ELIÇIN-ARIKAN, Décentralisation et urbanisme en Turquie, Thèse de doctorat n. r. en aménagement de l'espace et urbanisme, Université d'Aix-Marseille III, 1995, 474 p. en 2 vol.

32. Azerbaidjan SSR Atlasy (Atlas de la République Socialiste Soviétique d'Azerbaidjan, en turc azeri), Moscou, Administration centrale de géodésie et cartographie, 1979, 40 p. (planche p. 9) 